\begin{tabular}{|c|l|}
\hline Title & $\begin{array}{l}\text { Randomized phase II trial of first-line treatment with tail lored irinotecan and S-1 therapy versus S-1 monotherapy for } \\
\text { advanced or recurrent gastric carcinoma (JFMC31-0301) }\end{array}$ \\
\hline Author(s) & $\begin{array}{l}\text { Komatsu, Y oshito; Takahashi, Y Yutaka; Kimura, Y utaka; Oda, Hisashi; Tajima, Y usuke; Tamura, Shigey uki; Sakurai, } \\
\text { Jo; W akasugi, Takehiro; T atebe, Shigeru; Takahashi, Masahiro; Sakata, Y uh; Kitaji ima, Masaki; Sakamoto, Junichi; } \\
\text { Saji, Shigetoyo }\end{array}$ \\
\hline Citation & $\begin{array}{l}\text { Anti-Cancer Drugs, 22(6), 576-583 } \\
\text { https://doi.org/10.1097/CA D.0b013e328345b509 }\end{array}$ \\
\hline Issue Date & 2011-07 \\
\hline Doc URL & http://hdl.handle.net/2115/49475 \\
\hline Rights & Thisis a non-final version of an article published in final form in A nti-Cancer Drugs, Jul 2011, 22(6), 576-583 \\
\hline Type & article (author version) \\
\hline File Information & ACD22-6_576-583.pdf \\
\hline
\end{tabular}

Instructions for use 


\section{Randomized phase II trial of first-line treatment with tailored irinotecan plus S-1 therapy versus S-1 monotherapy for advanced or recurrent gastric carcinoma}

\section{(JFMC31-0301)}

Running head: Tailored IRIS vs. S-1 as first-line therapy for gastric cancer Yoshito Komatsu $^{\mathrm{a}}$, Yutaka Takahashi ${ }^{\mathrm{b}}$, Yutaka Kimura ${ }^{\mathrm{c}}$, Hisashi Oda ${ }^{\mathrm{d}}$, Yusuke Tajima ${ }^{\mathrm{e}}$, Shigeyuki Tamura ${ }^{\mathrm{f}}$, Jo Sakurai ${ }^{\mathrm{g}}$, Takehiro Wakasugi ${ }^{\mathrm{h}}$, Shigeru Tatebe ${ }^{\mathrm{i}}$, Masahiro Takahashi $^{\mathrm{j}}$, Yuh Sakata ${ }^{\mathrm{k}}$, Masaki Kitajima ${ }^{1}$, Junichi Sakamoto ${ }^{\mathrm{m}}$, and Shigetoyo Saji ${ }^{\mathrm{m}}$

a Division of Cancer Chemotherapy, Hokkaido University Hospital Cancer Centre, Sapporo, Japan

b Professor and Director of Cancer Chemotherapy, Kaken Hospital, International University of Health and Welfare, Ichikawa, Japan

c Dept. of Surgery, NTT West Osaka Hospital, Osaka, Japan

d Dept. of Internal Medicine, Kushiro Rosai Hospital, Kushiro, Japan

e Division of General \& Gastroenterological Surgery, Dept. of Surgery, School of Medicine, Showa University, Tokyo, Japan

f Dept. of Surgery, Kansai Rosai Hospital, Amagasaki, Japan

g Dept. of Gastroenterological Surgery, St. Marianna University School of Medicine, Kawasaki, Japan

h Dept. of Gastroenterological Surgery, Nagoya City University Graduate School of Medical Sciences, Nagoya, Japan

i Division of Surgical Oncology, Faculty of Medicine, Tottori University, Yonago, Japan

j Dept. of Surgery, Asahikawa Kosei Hospital, Asahikawa, Japan

k Department of Medical Oncology \& Internal Medicine, Misawa Municipal Hospital, Misawa, Japan

1 International University of Health and Welfare, Tokyo, Japan

m Japanese Foundation for Multidisciplinary Treatment of Cancer, Tokyo, Japan

\section{Corresponding Author:}

Yoshito KOMATSU, M.D., Ph.D. 
Division of Cancer Chemotherapy,

Hokkaido University Hospital Cancer Centre

Nishi-5, Kita-14, Kita-ku, Sapporo, Hokkaido 060-8638, Japan

Phone: +81-11-716-1161

Fax: +81-11-706-5657

E-mail: ykomatsu@med.hokudai.ac.jp 


\begin{abstract}
Objective: The pharmacokinetics of irinotecan vary markedly between individuals. This study sought to compare tailored irinotecan plus S-1 therapy with S-1 monotherapy for the treatment of patients with advanced/recurrent gastric cancer.
\end{abstract}

Methods: Patients with advanced/recurrent gastric cancer were randomized to receive tailored irinotecan plus S-1 (arm A) or S-1 alone (arm B). Arm A received S-1 (80-120 $\mathrm{mg} / \mathrm{m}^{2} /$ day) for 14 days, with irinotecan on days 1 and 15 . The initial irinotecan dose of $75 \mathrm{mg} / \mathrm{m}^{2}$ (Level 0 ) was adjusted for toxicity during the previous course. In arm B, S-1 $(80-120 \mathrm{mg} /$ day) was administered alone for 28 days, followed by 14 days without therapy.

Results: Ninety-five patients were randomized (48 to arm A and 47 to arm B). The response rate of the primary tumor (Japanese criteria) was 25.0\% in arm A (12/48) and $14.9 \%$ in arm B (7/47), while the response rates according to Response Evaluation Criteria In Solid Tumors (RECIST) were 27.8\% (10/36) versus 21.9\% (7/32).

Hematological toxicity, anorexia, and diarrhea were significantly more common in arm A, but both arms had similar grade 3-4 toxicities.

Conclusion: These findings suggest the usefulness of tailored irinotecan plus S-1 therapy for gastric cancer.

Keywords: Gastric cancer, irinotecan, S-1, tailored chemotherapy 


\section{Introduction}

Irinotecan hydrochloride (irinotecan) and 5-fluorouracil (5-FU) derivatives differ in their mechanism of action, and the efficacy of combined therapy with these agents has been demonstrated in animal experiments $[1,2]$. The combination of irinotecan with S-1 (an oral derivative of 5-FU developed in Japan in the 1990s), irinotecan/S-1 (IRIS), has also been examined, particularly in recent years [3-5].

In phase I/II studies in advanced gastric cancer, although response rates of $44 \%$ [6] and $49 \%$ [7] and overall survival times of 207 and 250 days have been shown with S-1 monotherapy, higher response rates have been reported when used in combination with irinotecan [3-5]. Phase I/II clinical trials of IRIS therapy showed that the recommended dose of irinotecan is $80 \mathrm{mg} / \mathrm{m}^{2}$ for a weekly regimen and 80 or $125 \mathrm{mg} / \mathrm{m}^{2}$ for a fortnightly regimen, with the response rate being $50 \%, 54 \%$, and $50 \%$ in patients receiving $80 \mathrm{mg} / \mathrm{m}^{2}$ weekly, $80 \mathrm{mg} / \mathrm{m}^{2}$ fortnightly, and $125 \mathrm{mg} / \mathrm{m}^{2}$ fortnightly, respectively [3-5]. However, severe adverse events occurred even at low irinotecan doses, suggesting that special care needs to be taken during administration of this drug. In Japan, chemotherapy for gastric cancer (an extremely common tumor in this country) is often performed on an outpatient basis, hence a safe regimen is required.

The rationale for tailored chemotherapy, in which the dosage is varied according to each patient's response, is based on individual variations of drug metabolizing enzyme activity related to genetic polymorphisms that influence pharmacokinetics and also lead to individual differences of toxicity and efficacy. In brief, tailored therapy aims to limit toxicity, improve compliance, and therefore maintain treatment for as long as possible 
and prolong survival. The efficacy of tailored FEC therapy (5-FU, epirubicin, and cyclophosphamide) as postoperative adjuvant chemotherapy for breast cancer has been shown [8]. In Japan, Takahashi et al. administered tailored gemcitabine therapy to patients with pancreatic cancer, and found improvements in both symptoms and quality of life in $75 \%$ of subjects [9].

Importantly, advanced gastric cancer generally has a poor prognosis and a standard chemotherapy treatment that is better that continuous infusion of 5-FU (5FU-ci) has not been established in Japan. We have previously employed an IRIS regimen for ambulatory treatment of patients with advanced gastric cancer, as reported by Komatsu et al [4]. Although the recommended dose of irinotecan was set at $125 \mathrm{mg} / \mathrm{m}^{2}$ in that study, grade 3 adverse events still occurred at the lowest dose administered $\left(100 \mathrm{mg} / \mathrm{m}^{2}\right)$. In the present randomized, phase II study, therefore, we set the initial dose of irinotecan at 75 $\mathrm{mg} / \mathrm{m}^{2}$ (one dose level below $100 \mathrm{mg} / \mathrm{m}^{2}$ ) in order to investigate the tolerability and survival benefit of tailored IRIS therapy compared with S-1 monotherapy for the ambulatory treatment of gastric cancer. The study was also designed to determine the best candidate for, and feasibility of, conducting a phase III comparative trial with 5FU-ci [10, $11]$.

\section{Materials and methods}

\section{Patients}

The protocol for this study has been reported previously [11], and an update is provided here. The subjects enrolled in this phase II study at Hokkaido University Graduate School of Medicine (Internal Medicine, Gastroenterology and Hematology), Sapporo Medical 
University School of Medicine (Surgery I), Hokkaido Cancer Center (Gastroenterology) Sapporo Social Insurance General Hospital, Sapporo Memorial Hospital of Surgery, Hokkaido Gastroenterology Hospital, Nikko Memorial Hospital, Asahikawa Kosei Hospital, Kushiro Rosai Hospital (Internal Medicine), Hirosaki University School of Medicine (Surgery II), Iwate Medical University(Surgery I), Senseki Hospital, Chiba Cancer Center (Clinical Oncology), Showa University School of Medicine (Surgery II), Kitasato Institute Hospital (Surgery), Tokyo Medical University St. Marianna University School of Medicine (Gastroenterological Surgery), Kanazawa University School of Medicine (Surgical Oncology), Fukui Red Cross Hospital, Gifu Municipal Hospital (Surgery), Ogaki Municipal Hospital (Surgery), Aichi Cancer Center (Gastroenterological Surgery), Nagoya City University Graduate School of Medical Sciences (Gastroenterological Surgery), NTT West Osaka Hospital, Osaka City University Graduate School of Medicine (Surgical Oncology), Saiseikai Senri Hospital, Osaka Medical College (General and Gastroenterological Surgery), Osaka Minami National Hospital, Hyogo Prefectural Nishinomiya Hospital, Kansai Rosai Hospital, Tottori University Faculty of Medicine (Surgical Oncology), Hiroshima University Research Institute for Radiation Biology and Medicine (Surgical Oncology), or Yamaguchi University School of Medicine (Digestive Surgery and Surgical Oncology) fulfilled the following criteria: (1) they had histologically or cytologically proven gastric cancer, (2) curative resection was impossible or the cancer was recurrent, (3) measurable or assessable lesions, (4) no radiation therapy or prior chemotherapy (adjuvant therapy with a 5-FU derivative and methotrexate, leucovorin, or low-dose cisplatin was allowed provided that it had been ceased at least 28 days before enrollment in the present study), 
(5) aged from 20-80 years, (6) expected survival time $\geq 12$ weeks, (7) Eastern Cooperative Oncology Group (ECOG) performance status of 0 or 1, (8) no severe dysfunction of major organs (bone marrow, heart, lungs, liver, and kidneys), and (9) provided written informed consent. The protocol was approved by the Institutional Review Board at each site at which the study was conducted and the Japanese Foundation for Multidisciplinary Treatment of Cancer.

\section{Randomization}

Patients were allocated to study arm A or B by the minimization method using the following factors for stratification: unresectable gastric cancer versus recurrent gastric cancer with adjuvant chemotherapy versus recurrent gastric cancer without adjuvant chemotherapy; well differentiated versus poorly-differentiated cancer; and institution (Fig. $1)$.

\section{Treatment schedule}

\section{(1) Tailored IRIS therapy (arm A)}

Irinotecan was administered at an initial dose of $75 \mathrm{mg} / \mathrm{m}^{2}$ as an intravenous (IV) infusion on days 1 and 15 of a 28-day cycle. S-1 was administered orally, with the initial dose being set at $40-60 \mathrm{mg} / \mathrm{m}^{2}$. It was administered twice daily for 14 days, followed by a 14-day withdrawal period to complete one cycle.

In subsequent cycles, the doses of these drugs were varied according to the most severe adverse events during the preceding cycle (Tables 1 and 2).

\section{(2) S-1 monotherapy (arm B)}

S-1 was administered orally, with the initial dose being set at $40-60 \mathrm{mg} / \mathrm{m}^{2}$. It was given 
twice daily for 28 days (day 1 to day 28), followed by a 14-day withdrawal period to complete one cycle (42 days in total).

In subsequent cycles, the dose was varied according to the most severe adverse events during the preceding cycle by the same dose reduction schedule as that used in arm A (Tables 1 and 2).

In patients from either arm, treatment was continued until progression occurred. Patients were also withdrawn from the study if their adverse events met the specified criteria or if they refused further treatment.

\section{Outcome measures}

The primary endpoint was the antitumor activity of each regimen, which was evaluated according to the Japanese Rules for Assessment of Gastric Carcinoma (13th Version) [12] and the internationally recognized Response Evaluation Criteria In Solid Tumors (RECIST) from the Guidelines for Evaluation of the Response to Treatment in Solid Tumors [13]. The response rate was determined as the percentage of patients with either a complete response (CR) or a partial response (PR).

Secondary endpoints were adverse events, time to treatment failure (TTF), time to progression (TTP), and overall survival. Adverse events were graded according to the National Cancer Institute (NCI) Common Toxicity Criteria (CTC) (version 2) [14].

\section{Statistical Analysis}

The target number of patients was determined by the method used in the randomized phase II clinical trials conducted by Simon et al. [10], i.e., the number of patients per 
group required to select the best treatment with a probability $\geq 90 \%$ based on an assumed difference of the response rate between the baseline and best treatments. Assuming that the response rate was $40 \%$ for S-1 monotherapy [7] and 55\% for IRIS therapy [5], 37 patients per group would be required. Considering the possible enrollment of ineligible patients, the number per group was set at 45 .

For patient background data, percentages were calculated and intergroup differences were assessed by Fisher's exact test or the $\chi 2$-test for continuous variables and by Wilcoxon's rank sum test for discrete variables. Adverse events were graded according to severity, and intergroup comparison was done by Wilcoxon's rank sum test. Intergroup comparison of the response rate was performed by Fisher's exact test. For the TTF, TTP, and overall survival, probability curves were drawn by the Kaplan-Meier method and comparison between the two arms was performed by the log-rank test. Cessation of therapy, tumor progression, and death were used to determine the TTF, while tumor progression and death were used to define the TTP.

The level of significance was $P=0.15$ for analysis of background factors and $P=0.05$ for other analyses. Statistical analysis was carried out with SAS version 9.1 software.

\section{Results}

\section{Enrolment and follow-up}

Patients were enrolled from August 2003 to March 2005. The follow-up period was set at two years, commencing from the completion of patient enrollment, and final follow-up 
was conducted in April 2007.

\section{Patient profile}

All of the 95 patients enrolled were eligible. Two patients in arm A did not receive any treatment because of refusal to start therapy (the patient switched to another hospital) and deterioration of the general condition in one case each. All 95 eligible patients were included in the analyses, except that the two untreated patients were excluded from safety evaluation (Fig. 1). When background factors were assessed, age showed a bias between the two arms $(P=0.022$, Wilcoxon's rank sum test $)$. Patients aged from $71-80$ years accounted for $45.8 \%$ of $\operatorname{arm} \mathrm{A}$, and this treatment arm was older than $\operatorname{arm} \mathrm{B}$. However, the two arms were well matched with respect to the other background factors (Table 3).

\section{Treatment}

The median number of cycles of chemotherapy was 3 (range: 0-15) in arm A and 2 (range: 1-12) in arm B. The median duration of treatment was 84.5 days (95\% CI: 65-99 days) in arm A and 92 days (95\% CI: 64-126 days) in arm B, showing no difference. The reasons for ceasing therapy in arm A included tumor progression in $47.9 \%$, adverse events in $27.1 \%$, and other reasons in $25.0 \%$ of patients, while the corresponding values for arm B were $66.0 \%, 23.4 \%$, and $10.6 \%$. The percentage of patients ceasing treatment due to adverse events was similar in both arms. In arm A, only two patients stopped therapy due to grade 3 or 4 diarrhea. The frequency of S-1 dose reduction did not differ between the two arms. Reduction of the irinotecan dose was performed in $39.1 \%$ of patients in arm A, while the dose was increased in $30.4 \%$ of patients. Dose reduction of irinotecan was undertaken for hematological toxicity in $21.7 \%$, diarrhea in $10.9 \%$, and 
other symptoms in $15.2 \%$ of patients.

In arm A, eight of the 46 patients started irinotecan at dose level 0 and then received a higher dosage (dose level +1$)$ in the second cycle, while 19 patients stayed at dose level 0 and 12 patients had a reduction of one level (dose level -1$)$. By the third cycle, the 46 patients were distributed from dose level -2 to dose level +2 (Fig. 2). In contrast, no patient in arm B needed reduction of the dose to level -2 .

\section{Antitumor effect}

Overall evaluation was done according to the Japanese rules for gastric carcinoma (Japanese Gastric Cancer Association, 1998), including evaluation of the primary tumor and RECIST for evaluation of measurable metastases [13]. According to the Japanese criteria, 12 patients from arm A (including two with $\mathrm{CR}$ ) showed a response (CR or PR) and the response rate was $25.0 \%$, while seven patients from arm B (including one with CR) showed a response and the response rate was $14.9 \%$. According to RECIST, the response rate was $27.8 \%$ in arm A and $21.9 \%$ in arm B. Among the eligible patients, seven from arm A and three from arm B withdrew during the first cycle owing to adverse events, complications, or patient request. Two patients each in arms A and B had incomplete data for other reasons. All of these patients were classified as unevaluable. When they were excluded, the response rate was $30.8 \%$ in arm A and $16.7 \%$ in arm B according to the Japanese criteria, while the corresponding RECIST rates were $33.3 \%$ and $24.1 \%$ (Table 4$)$.

To assess whether dose modification of irinotecan (tailored therapy) influenced the antitumor effect of therapy in arm A, the response rate over three cycles was determined 
for each dose level in patients receiving at least three cycles of treatment. As a result, no influence of the different irinotecan dosages was noted (Table 5).

\section{Adverse events}

Common grade 3 or 4 adverse events were a decreased neutrophil count (23.9\%), anorexia (17.4\%), decreased hemoglobin (10.9\%), and fatigue/malaise (10.9\%) in arm A, while a decreased neutrophil count (12.8\%) and anorexia (10.6\%) were common in arm B. The only grade 4 adverse events were anorexia in one patient from arm A, and a decreased white blood cell count/neutrophil count and vomiting in one patient each from arm B (Table 6).

\section{Outcome}

The median TTF was 82 days in arm A (95\% CI: 60-105 days) and 73 days in arm B (95\% CI: 59-113 days), while the median TTP was 148 days (95\% CI: 97-210 days) and 115 days (95\% CI: 59-168 days), respectively. Both endpoints showed no significant difference between the two arms $(\mathrm{P}=0.855, P=0.214)$. The median survival time (MST) was 276 days in arm A (95\% CI: 210-393 days) and 373 days in arm B (95\% CI: 305-523 days), and there was also no significant difference in overall survival $(P=0.203)$. Because the number of patients aged $\geq 71$ years showed a bias between the arms, MST was calculated separately for patients aged $\leq 70$ years; it was 280 days in arm A (95\% CI: 192-424 days) and 321 days in arm B (95\% CI: 270-451 days), showing no significant difference $(\mathrm{P}=0.874)$. 


\section{Discussion}

Development of a "patient-friendly treatment" is one of the main goals of the Japanese Foundation for Multidisciplinary Treatment of Cancer. Accordingly, the present study investigated tailored therapy with irinotecan, which shows marked interindividual variability in the response to treatment at each dose causing adverse effects, and demonstrated manageable toxicity and improved clinical response with tailored IRIS compared with S-1 monotherapy, suggesting tailored IRIS therapy is more promising for a phase III trial.

It is noteworthy that tailored therapy did not cause any grade 4 hematological toxicity, while grade 4 non-hematological toxicity was limited to anorexia in one patient. Because grade 3 or 4 toxicities accounted for over $35 \%$ of all toxicities even at an initial irinotecan dose of $75 \mathrm{mg} / \mathrm{m}^{2}$, many patients would presumably have suffered from grade 4 toxicity if the starting dose had been $125 \mathrm{mg} / \mathrm{m}^{2}$, which was the recommended dose according to the phase I/II trials. Therefore, tailored IRIS therapy achieved a considerable reduction of risk. When the relationship between the irinotecan dosage and tumor response was assessed in the third cycle, no difference of the response rate was found between the dose levels (although there were small numbers in each dose group). These findings indicate that tailored therapy not only reduces risk, but also sets the appropriate dose for each individual patient. Our results are in agreement with the report of a pilot study of tailored gemcitabine therapy for pancreatic cancer performed by Takahashi et al [15].

In the present trial, the respective response rates with tailored IRIS therapy were greater than with S-1 monotherapy, $25.0 \%$ and $14.9 \%$ according to Japanese criteria, and $27.8 \%$ and $21.9 \%$ according to RECIST; however, these response rates were lower compared 
with the results of similar clinical studies (about 50\%) and only survival was longer in the S-1 monotherapy arm. Nevertheless, it is difficult to make a direct comparison of response rates between the present trial and other studies. The low response rates in the present study may have reflected the enrollment of patients who were not highly selected. In addition, survival may have been influenced by the age bias among the arms, since patients aged $\geq 71$ years accounted for $45.8 \%(n=22)$ of arm A versus $14.9 \%(n=7)$ of arm $\mathrm{B}$, even though this was a randomized trial. In fact, when analysis was conducted after excluding patients aged 71 or older, the survival time was similar for both arms.

The results of a phase III trial of IRIS versus S-1 monotherapy (study GC0301/TOP-002), which used a different IRIS regimen from that employed in the present trial (the irinotecan dose being much lower in the present study), were reported at the 2008 Gastrointestinal Cancer Symposium [16]. In that study, despite the lack of a statistically significant difference in overall survival between IRIS and S-1 alone, the MST of 12.8 months achieved with IRIS was longer than the 10.5 months achieved with S-1, and was comparable to the 13 months achieved by standard therapy with cisplatin plus S-1 in Japan (SPIRITS trial) [17].

In both study GC0301/TOP-002 and the present trial, S-1 monotherapy was used as the control and no significant difference in survival time was observed between the IRIS and control arms, suggesting that low doses of irinotecan may have led to the lack of a significant difference from the control group in both studies. The starting doses of irinotecan in study GC0301/TOP-002 and the present trial were only $160 \mathrm{mg} / \mathrm{m}^{2}$ over 5 weeks and $150 \mathrm{mg} / \mathrm{m}^{2}$ over 4 weeks, respectively, which means weekly doses of only 32 $\mathrm{mg} /{ }^{2}$ and $37.5 \mathrm{mg} / \mathrm{m}^{2}$. These are approximately half of the weekly dose $\left(62.5 \mathrm{mg} / \mathrm{m}^{2}\right)$ 
delivered by the original IRIS regimen used as the model for the present trial.

There are three major problems with tailored chemotherapy: 1) selection of the starting dose, 2) selection of the dose modification method, and 3) the risk of undertreatment with the key drug. The starting dose of irinotecan for the present trial was set at $75 \mathrm{mg} / \mathrm{m}^{2}$ based on the results of a phase I/II study performed by Komatsu et al. [4], who reported grade 4 myelosuppression at $125 \mathrm{mg} / \mathrm{m}^{2}$ (the recommended dose) and also at $100 \mathrm{mg} / \mathrm{m}^{2}$. Despite this low starting dose in the present study, 16 of the 46 patients (35\%) withdrew from treatment or needed a dose reduction in the next cycle owing to grade 3 or 4 toxicity. In contrast, a dose increase after grade 0 or 1 toxicity was only possible in 8 patients $(17 \%)$. On the other hand, the final dose showed a normal distribution centering around dose level 0 in the 25 patients (54\%) who could be assessed. Furthermore, among the eight patients receiving at least eight cycles of treatment, three achieved an increase over the starting dose, two had a decrease, and three had no change, i.e., the final dose showed a wide dispersion. The criterion for dose reduction was grade 2 toxicity. Of the 46 patients in arm A, 19 had grade 2 toxicity and the same dose of $75 \mathrm{mg} / \mathrm{m}^{2}$ was administered in the next cycle. None of these 19 patients achieved a dose increase during the cycle after that. Instead, five of them needed dose reduction or withdrew from treatment. These results suggest that the use of dose-limiting toxicity to set the starting dose and for dose increase/reduction is open to question.

With the use of gemcitabine or taxanes, most severe toxicities are hematological, whereas the dose-limiting toxicities for irinotecan are generally diarrhea, vomiting, and other nonhematological events (e.g., anorexia and malaise). Hematological events are easy to monitor objectively, but many non-hematological events cannot be assessed objectively, 
and this can lead to problems when modifying the dosage based on toxicities (as was done in the present study).

Thirdly, when the doses of anticancer agents are set at levels that will only induce mild adverse reactions, the response to treatment may be reduced. Most clinical trials of chemotherapy agents attempt to maximize efficacy by using the maximum tolerated dose determined in a phase I trial. Therefore, the low response rate and the short survival time obtained in the present trial of a low-dose regimen need to be compared with data from other clinical trials, whilst bearing in mind the dosage differences.

In conclusion, the results of the present randomized phase II trial showed manageable tolerability and improved efficacy with tailored IRIS therapy compared with S-1 monotherapy suggesting IRIS is more promising for a phase III trial. However, if a phase III trial is to be designed to achieve the maximum clinical efficacy, it would be difficult to conduct a controlled trial with an arm for tailored IRIS therapy. It might be possible to perform a tailored-dose trial after the best standard therapy has been determined by an ordinary phase III trial. 


\section{Acknowledgements}

Financial support for this study was provided by the Japanese Foundation for Multidisciplinary Treatment of Cancer. The authors thank Nila Bhana of inScience Communications, a Wolters Kluwer business, who provided assistance with Englishlanguage editing. The authors have no conflicts of interest that are directly relevant to the content of this study. 


\section{References}

1 Funakoshi S, Aiba K, Shibata H, Mizunama N, Hirano A, Ogihara A, et al. Enhanced antitumor activity of SN-38, an active metabolite of CPT-11, and 5fluorouracil combination for human colorectal cancer cell lines. Proc Am Soc Clin Oncol 1993; 193:abstr 563.

2 Fukushima M, Fujioka A, Uchida J, Nakagawa F, Takechi T. Thymidylate synthase (TS) and ribonucleotide reductase (RNR) may be involved in acquired resistance to 5-fluorouracil (5-FU) in human cancer xenografts in vivo. Eur J Cancer 2001; 37:1681-1687.

3 Narahara H, Takiuchi H, Tsujinaka T, Furukawa H, Tsukuma H, Taguchi H. Phase I study of CPT-11 plus S-1 in patients with metastatic gastric cancer. Proc Am Soc Clin Oncol 2002; 21:170 abstr 677.

4 Komatsu y, Takeda H, Takei M, Kato T, Tateyama M, Miyagishima T. Phase I and PK study of S-1 and irinotecan (CPT-11) in patients with advanced gastric cancer (AGC). Proc Am Soc Clin Oncol 2002; 21:171 abstr 683.

5 Inokuchi M, Yamashita T, Yamada H, Kojima K, Ichikawa W, Nihei Z, et al. Phase I/II study of S-1 combined with irinotecan for metastatic advanced gastric cancer. Br J Cancer 2006; 94:1130-1135.

6 Koizumi W, Kurihara M, Nakano S, Hasegawa K. Phase II study of S-1, a novel oral derivative of 5-fluorouracil, in advanced gastric cancer. For the S-1 Cooperative Gastric Cancer Study Group. Oncology 2000; 58:191-197.

7 Sakata Y, Ohtsu A, Horikoshi N, Sugimachi K, Mitachi Y, Taguchi T. Late phase II study of novel oral fluoropyrimidine anticancer drug S-1 (1 M tegafur- $0.4 \mathrm{M}$ gimestat$1 \mathrm{M}$ otastat potassium) in advanced gastric cancer patients. Eur J Cancer 1998; 34:17151720 .

8 Bergh J, Wiklund T, Erikstein B, Lidbrink E, Lindman H, Malmstrom P, et al. Tailored fluorouracil, epirubicin, and cyclophosphamide compared with marrowsupported high-dose chemotherapy as adjuvant treatment for high-risk breast cancer: a randomised trial. Scandinavian Breast Group 9401 study. Lancet 2000; 356:1384-1391. 9 Takahashi Y, Mai M, Sawabu N, Nishioka K. A pilot study of individualized maximum repeatable dose (iMRD), a new dose finding system, of weekly gemcitabine for patients with metastatic pancreas cancer. Pancreas 2005; 30:206-210.

10 Simon R, Wittes RE, Ellenberg SS. Randomized phase II clinical trials. Cancer Treat Rep 1985; 69:1375-1381.

11 Takahashi Y, Sakamoto J, Takeuchi T, Mai M, Kubota T, Kitajima M, et al. A randomized phase II clinical trial of tailored CPT-11 + S-1 vs S-1 in patients with advanced or recurrent gastric carcinoma as the first line chemotherapy. Jpn J Clin Oncol 2004; 34:342-345.

12 Japanese Gastric Cancer Association. Japanese classification of gastric carcinoma - 2nd English Edition. Gastric Cancer 1998; 1:10-24.

13 Therasse P, Arbuck SG, Eisenhauer EA, Wanders J, Kaplan RS, Rubinstein L, et al. New guidelines to evaluate the response to treatment in solid tumors. European Organization for Research and Treatment of Cancer, National Cancer Institute of the United States, National Cancer Institute of Canada. J Natl Cancer Inst 2000; 92:205-216. 
14 Trotti A, Byhardt R, Stetz J, Gwede C, Corn B, Fu K, et al. Common toxicity criteria: version 2.0. an improved reference for grading the acute effects of cancer treatment: impact on radiotherapy. Int J Radiat Oncol Biol Phys 2000; 47:13-47. 15 Takahashi Y, Yamashita K, Kitakata H, Mai M. A new dose finding system in chemotherapy for solid tumors -individualized maximum repeatable dose (iMRD) of weekly gemcitabine for metastatic pancreas cancer. Proc Am Soc Clin Oncol 2003; 22:303 abstr 1217.

16 Imamura H, Ilishi H, Tsuburaya A, Hatake K, Imamoto H, Esaki T, et al. Randomized phase III study of irinotecan plus S-1 (IRIS) versus S-1 alone as first-line treatment for advanced gastric cancer (GC0301/TOP-002). Presented at the 2008 Gastrointestinal Cancers Symposium, Orlando, FL, January 15-17, 2008; 2008. p. abstr 5. 17 Koizumi W, Narahara H, Hara T, Takagane A, Akiya T, Takagi M, et al. S-1 plus cisplatin versus S-1 alone for first-line treatment of advanced gastric cancer (SPIRITS trial): a phase III trial. Lancet Oncol 2008; 9:215-221. 
Table 1 Dose modification of irinotecan and S-1

\begin{tabular}{|c|c|c|c|c|c|c|}
\hline & \multicolumn{2}{|c|}{ Escalation } & \multicolumn{2}{|c|}{ No change } & \multicolumn{2}{|c|}{ Reduction } \\
\hline & Irinotecan & S-1 & Irinotecan & S-1 & Irinotecan & S-1 \\
\hline Hematological toxicity ${ }^{a}$ & Gr. 0-1 & - & Gr. 2 & Gr. 0-2 & \multicolumn{2}{|c|}{ Gr. 3-4 } \\
\hline $\begin{array}{l}\text { Symptoms/signs }{ }^{a} \\
\text { (excluding nausea and } \\
\text { vomiting) }\end{array}$ & Gr. 0-1 & - & Gr. 2 & Gr. 0-2 & \multicolumn{2}{|r|}{ Gr. 3} \\
\hline Diarrhea $^{a}$ & Gr. 0 & - & Gr. 1 & - & Gr. 2 & - \\
\hline Serum creatinine & - & - & - & $\leq \mathrm{ULN}$ & - & $\leq \mathrm{ULN} \times 1.1-1.5$ \\
\hline Others & - & - & - & - & $\begin{array}{l}\text { Skip the } \\
\text { second } \\
\text { dose }\end{array}$ & - \\
\hline
\end{tabular}

a Graded according to the National Cancer Institute Common Toxicity Criteria (version 2) [13]. Gr, grade; ULN, upper limit of normal. 
Table 2 Dose levels of irinotecan and S-1

\begin{tabular}{|c|c|c|c|c|}
\hline \multirow{3}{*}{ Irinotecan } & & \multicolumn{3}{|c|}{ S-1 } \\
\hline & & \multicolumn{3}{|c|}{ Body Surface Area } \\
\hline & & $<1.25 \mathrm{~m}^{2}$ & $\geq 1.25 \mathrm{~m}^{2}-<1.5 \mathrm{~m}^{2}$ & $\geq 1.5 \mathrm{~m}^{2}$ \\
\hline $125 \mathrm{mg} / \mathrm{m}^{2}$ & $\uparrow$ & - & - & - \\
\hline $125 \mathrm{mg} / \mathrm{m}^{2}$ & Level +2 & - & - & - \\
\hline $100 \mathrm{mg} / \mathrm{m}^{2}$ & Level +1 & - & - & - \\
\hline $75 \mathrm{mg} / \mathrm{m}^{2}$ & Starting dosage & $40 \mathrm{mg} \times 2$ & $50 \mathrm{mg} \times 2$ & $60 \mathrm{mg} \times 2$ \\
\hline $50 \mathrm{mg} / \mathrm{m}^{2}$ & Level -1 & $25 \mathrm{mg} \times 2$ & $40 \mathrm{mg} \times 2$ & $50 \mathrm{mg} \times 2$ \\
\hline $25 \mathrm{mg} / \mathrm{m}^{2}$ & Level -2 & Discontinue & $25 \mathrm{mg} \times 2$ & $40 \mathrm{mg} \times 2$ \\
\hline Discontinue & Level -3 & - & Discontinue & $25 \mathrm{mg} \times 2$ \\
\hline- & Level -4 & - & - & Discontinue \\
\hline
\end{tabular}


Table 3 Patient characteristics

\begin{tabular}{cccccccc} 
& \multicolumn{2}{c}{ Arm A } & \multicolumn{2}{c}{ Arm B } & Total & $P$ value \\
\hline Total & 48 & $(100 \%)$ & 47 & $(100 \%)$ & 95 & $(100 \%)$
\end{tabular}

Diagnosis

$\begin{array}{lccccccc}\text { Unresectable } & 33 & (68.8 \%) & 33 & (70.2 \%) & 66 & (69.5 \%) & 0.85(\mathrm{C}) \\ \text { Recurrence (with Adj.) } & 9 & (18.8 \%) & 7 & (14.9 \%) & 16 & (16.8 \%) \\ \text { Recurrence (without Adj.) } & 6 & (12.5 \%) & 7 & (14.9 \%) & 13 & (13.7 \%)\end{array}$

Histology

$\begin{array}{lccccccc}\text { Well-differentiated } & 22 & (45.8 \%) & 20 & (42.6 \%) & 42 & (44.2 \%) & 0.81(\mathrm{C}) \\ \text { Poorly-differentiated } & 25 & (52.1 \%) & 25 & (53.2 \%) & 50 & (52.6 \%) \\ \text { Others } & 1 & (2.1 \%) & 2 & (4.3 \%) & 3 & (3.2 \%)\end{array}$

BSA (daily dose of S-1)

$\begin{array}{lccccccc}<1.25 \mathrm{~m}^{2}(80 \mathrm{mg}) & 3 & (6.3 \%) & 1 & (2.1 \%) & 4 & (4.2 \%) & 0.58(\mathrm{C}) \\ \geq 1.25-<1.5 \mathrm{~m}^{2}(100 \mathrm{mg}) & 19 & (39.6 \%) & 18 & (38.3 \%) & 37 & (38.9 \%) & 0.48(\mathrm{~W}) \\ \geq 1.5 \mathrm{~m}^{2}(120 \mathrm{mg}) & 26 & (54.2 \%) & 28 & (59.6 \%) & 54 & (56.8 \%) & \end{array}$

Sex

$\begin{array}{llllllll}\text { Male } & 34 & (70.8 \%) & 37 & (78.7 \%) & 71 & (74.7 \%) & 0.48(\mathrm{~F}) \\ \text { Female } & 14 & (29.2 \%) & 10 & (21.3 \%) & 24 & (25.3 \%) & \end{array}$

Age

$\begin{array}{lccccccc}20-50 & 4 & (8.3 \%) & 9 & (19.1 \%) & 13 & (13.7 \%) & 0.005(\mathrm{C}) \\ 51-60 & 10 & (20.8 \%) & 9 & (19.1 \%) & 19 & (20.0 \%) & 0.02(\mathrm{~W}) \\ 61-70 & 12 & (25.0 \%) & 22 & (46.8 \%) & 34 & (35.8 \%) & \\ 71-80 & 22 & (45.8 \%) & 7 & (14.9 \%) & 29 & (30.5 \%) \\ \text { Range } & & 47-78 & 24-76 & 24-78 \\ \text { Median } & & 70 & 63 & 66\end{array}$

ECOG PS 


$\begin{array}{llllllll}0 & 38 & (79.2 \%) & 35 & (74.5 \%) & 73 & (76.8 \%) & 0.63(\mathrm{~F}) \\ 1 & 10 & (20.8 \%) & 12 & (25.5 \%) & 22 & (23.2 \%) & \end{array}$

\section{Prior treatment}

\begin{tabular}{lllllll} 
Surgery & 2 & $(4.2 \%)$ & 4 & $(8.5 \%)$ & 6 & $(6.3 \%)$ \\
Others & 1 & $(2.1 \%)$ & 0 & $(0.0 \%)$ & 1 & $(1.1 \%)$ \\
\hline
\end{tabular}

Adj, adjuvant therapy; BSA, body surface area; $C, \chi^{2}$ test; ECOG, Eastern Cooperative Oncology Group; PS, performance status; W, Wilcoxon test; F, Fisher's exact test 


\begin{tabular}{|c|c|c|c|c|c|c|c|}
\hline & \multicolumn{2}{|c|}{ Arm A } & \multicolumn{2}{|c|}{ Arm B } & \multicolumn{2}{|c|}{ Total } & $P$ value \\
\hline \multicolumn{8}{|l|}{ JCGC } \\
\hline CR & 2 & $(4.2 \%)$ & 1 & $(2.1 \%)$ & 3 & $(3.2 \%)$ & \\
\hline PR & 10 & $(20.8 \%)$ & 6 & $(12.8 \%)$ & 16 & $(16.8 \%)$ & $0.11(W)$ \\
\hline NC & 17 & $(35.4 \%)$ & 19 & $(40.4 \%)$ & 36 & $(37.9 \%)$ & $\begin{array}{l}\text { (excludes } \\
\text { NE cases) }\end{array}$ \\
\hline PD & 10 & $(20.8 \%)$ & 16 & $(34.0 \%)$ & 26 & $(27.4 \%)$ & \\
\hline $\mathrm{NE}$ & 9 & $(18.8 \%)$ & 5 & $(10.6 \%)$ & 14 & $(14.7 \%)$ & \\
\hline Total & 48 & $(100.0 \%)$ & 47 & $(100.0 \%)$ & 95 & $(100.0 \%)$ & \\
\hline $\mathrm{R}(\mathrm{CR}+\mathrm{PR})$ & $12 / 48$ & $(25.0 \%)$ & $7 / 47$ & (14.9\%) & $19 / 95$ & $(20.0 \%)$ & $0.30(F)$ \\
\hline $\begin{array}{l}\text { (excludes NE } \\
\text { cases) }\end{array}$ & $12 / 39$ & $(30.8 \%)$ & $7 / 42$ & $(16.7 \%)$ & $19 / 81$ & $(23.5 \%)$ & $0.19(F)$ \\
\hline
\end{tabular}

\section{RECIST}

$\begin{array}{lccccccc}\text { CR } & 1 & (2.8 \%) & 1 & (3.1 \%) & 2 & (2.9 \%) & \\ \text { PR } & 9 & (25.0 \%) & 6 & (18.8 \%) & 15 & (22.1 \%) & 0.13(\mathrm{~W}) \\ \text { SD } & 14 & (38.9 \%) & 10 & (31.3 \%) & 24 & (35.3 \%) & \begin{array}{c}\text { (excludes } \\ \text { NE cases) }\end{array} \\ \text { PD } & 6 & (16.7 \%) & 12 & (37.5 \%) & 18 & (26.5 \%) & \\ \text { NE } & 6 & (16.7 \%) & 3 & (9.4 \%) & 9 & (13.2 \%) & \\ \text { Total } & 36 & (100 \%) & 32 & (100 \%) & 68 & (100 \%) & \\ & & & & & & & \\ \text { R (CR+PR) } & 10 / 36 & (27.8 \%) & 7 / 32 & (21.9 \%) & 17 / 68 & (25.0 \%) & 0.78(\mathrm{~F}) \\ \begin{array}{l}\text { (excludes NE } \\ \text { cases) }\end{array} & 10 / 30 & (33.3 \%) & 7 / 29 & (24.1 \%) & 17 / 59 & (28.8 \%) & 0.56(\mathrm{~F})\end{array}$

CR, complete response; F, Fisher's exact test; JCGC, Japanese Classification of Gastric Carcinoma; NC, no change; NE, not evaluable; PD, progressive disease; PR, partial response; R, response; RECIST, Response Evaluation Criteria In Solid Tumors; W, Wilcoxon test 
Table 5 Tumor response in Arm A stratified by the dose level of irinotecan ${ }^{a}$

\begin{tabular}{crll}
\hline Dose Level & \multicolumn{3}{c}{ Response } \\
\hline Level +2 & $1 / 4$ & $(25 \%)$ & \multicolumn{2}{c}{$9 / 22(40.9 \%)$} \\
Level +1 & $3 / 3$ & $(100 \%)$ & \\
Level 0 & $5 / 15$ & $(33.3 \%)$ & \\
Level -1 & $1 / 4$ & $(25 \%)$ & \\
Level -2 & $2 / 3$ & $(66.7 \%)$ & \\
\hline Total & $12 / 29$ & $(41.4 \%)$ & \\
\hline
\end{tabular}

a Patients who received more than 3 courses were evaluated 
Table 6 Grade 3 or 4 toxicities

\begin{tabular}{lccccc}
\hline & \multicolumn{2}{c}{ Arm a } & \multicolumn{2}{c}{ Arm B } & \multirow{2}{*}{ P value* } \\
\cline { 2 - 5 } & Gr. 3 & Gr. 4 & Gr. 3 & Gr. 4 & \\
\hline Anemia & $10.9 \%$ & $0 \%$ & $4.3 \%$ & $0 \%$ & 0.26 \\
Leucopenia & $8.7 \%$ & $0 \%$ & $2.1 \%$ & $2.1 \%$ & 0.43 \\
Neutropenia & $23.9 \%$ & $0 \%$ & $10.6 \%$ & $2.1 \%$ & 0.18 \\
Thrombocytopenia & $0.0 \%$ & $0 \%$ & $4.3 \%$ & $0 \%$ & 0.49 \\
Albumin $\downarrow$ & $4.3 \%$ & - & $0 \%$ & - & 0.24 \\
AST $\uparrow$ & $2.2 \%$ & $0 \%$ & $0 \%$ & $0 \%$ & 0.49 \\
ALP $\uparrow$ & $2.2 \%$ & $0 \%$ & $2.1 \%$ & $0 \%$ & 1.0 \\
Na $\downarrow$ & $6.5 \%$ & $0 \%$ & $0 \%$ & $0 \%$ & 0.11 \\
K $\downarrow$ & $4.3 \%$ & $0 \%$ & $2.1 \%$ & $0 \%$ & 0.61 \\
Stomatitis & $2.2 \%$ & $0 \%$ & $0 \%$ & $0 \%$ & 0.49 \\
Anorexia & $15.2 \%$ & $2.2 \%$ & $10.6 \%$ & $0 \%$ & 0.38 \\
Nausea & $6.5 \%$ & - & $6.4 \%$ & - & 1.0 \\
Vomiting & $2.2 \%$ & $0 \%$ & $2.1 \%$ & $2.1 \%$ & 1.0 \\
Diarrhea & $4.3 \%$ & $0 \%$ & $2.1 \%$ & $0 \%$ & 0.61 \\
Fatigue & $10.9 \%$ & $0 \%$ & $6.4 \%$ & $0 \%$ & 0.61 \\
\hline
\end{tabular}

* Fisher's exact test 


\section{Figure Legends}

Fig. 1 CONSORT diagram for the study. CPT-11, irinotecan hydrochloride; ITT, intentto-treat. *One patient switched to another hospital and the condition of the other patient deteriorated due to stenosis.

Fig. 2 Pattern of changes in the dose level of irinotecan (1st -4 th courses) and final dose level in arm A 


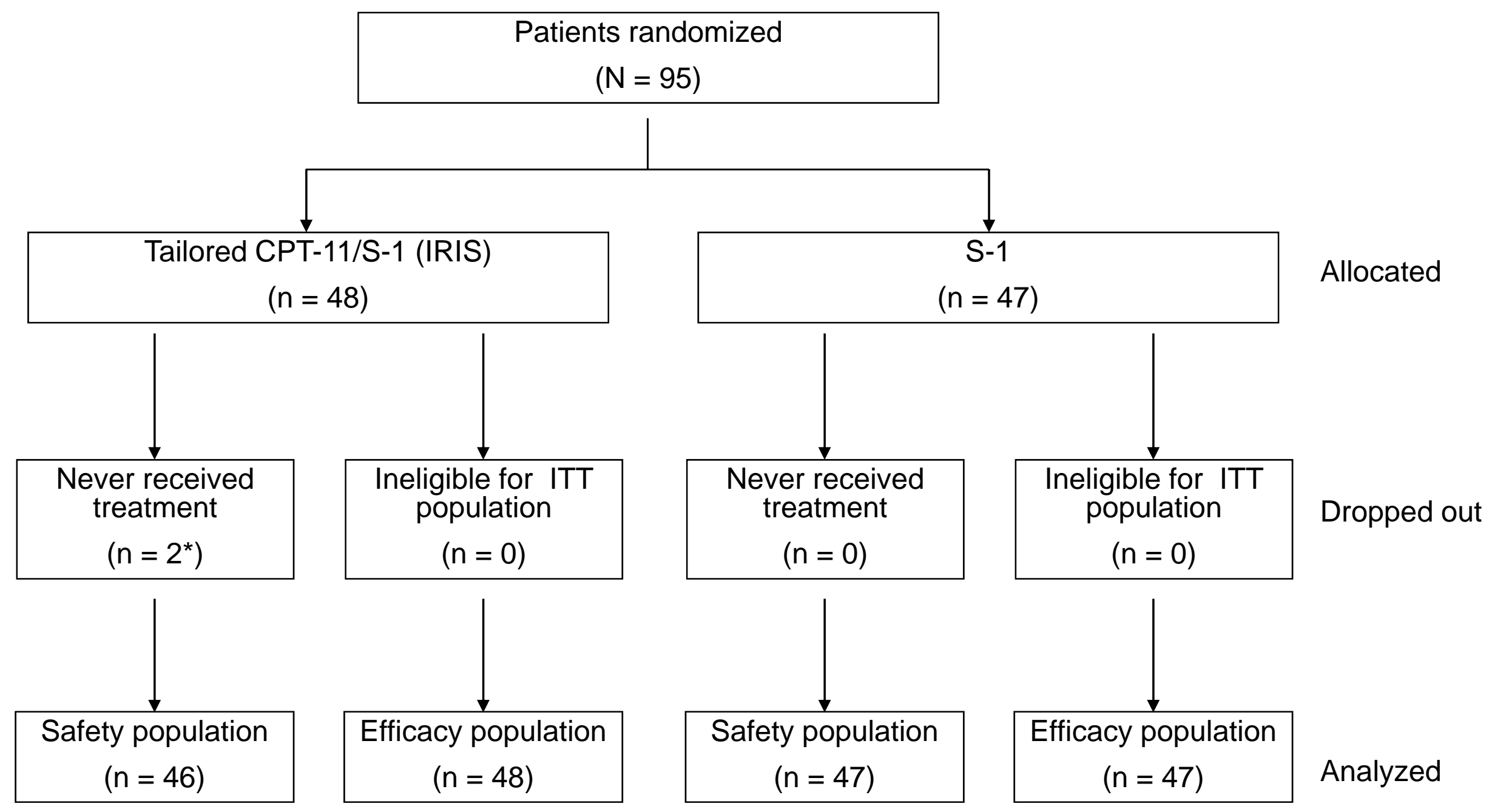



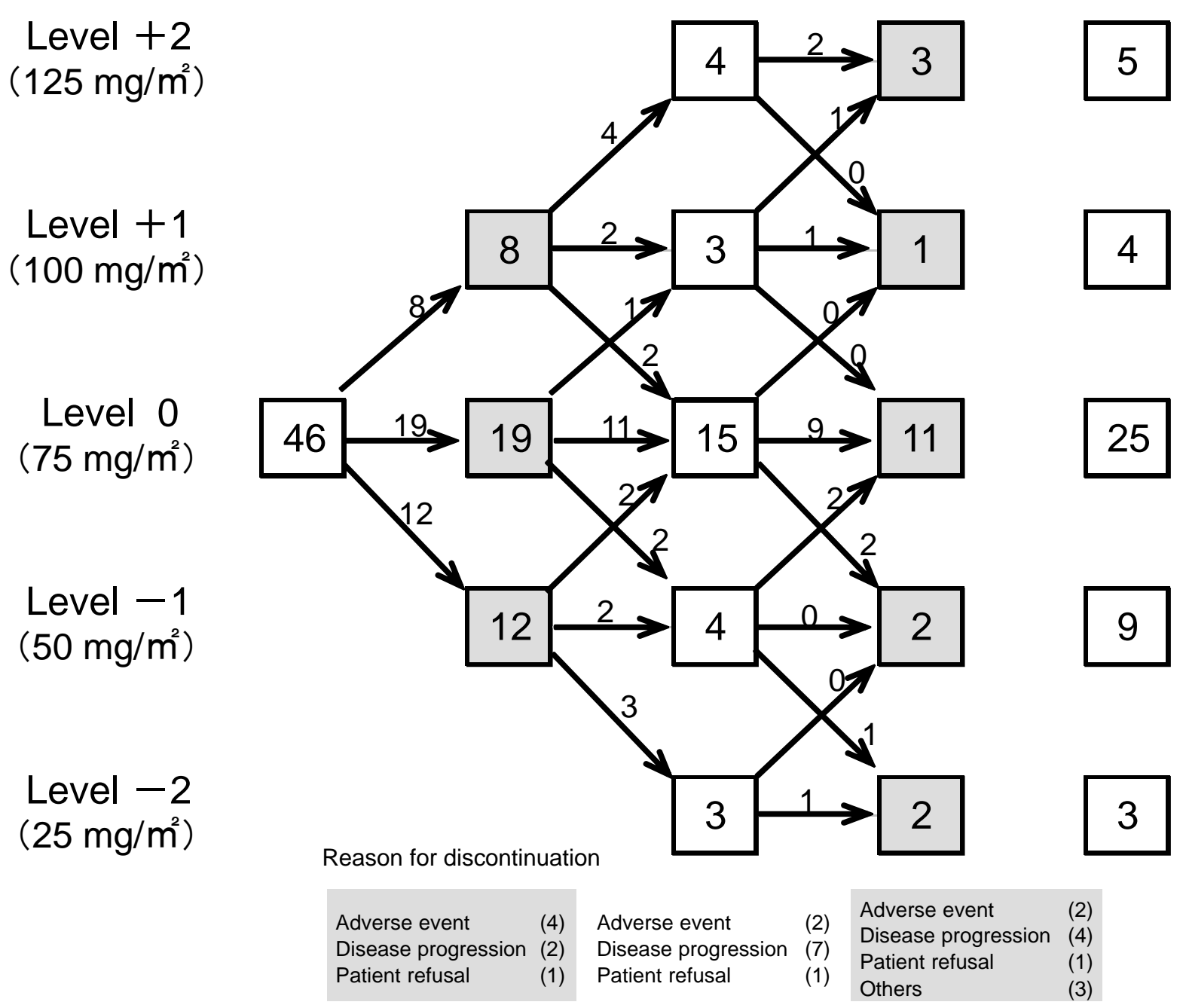